\title{
BMJ Open Protocol for development of a core outcome set for clinical trials in melasma
}

\author{
Sarah A Ibrahim, ${ }^{1}$ Bianca Y Kang, ${ }^{1}$ Daniel I Schlessinger, ${ }^{2}$ Sarah G Chiren, ${ }^{1}$ \\ Jennifer C Tang, ${ }^{3}$ Jamie J Kirkham (D) , ${ }^{4}$ Jochen Schmitt, ${ }^{5}$ Emily Poon, ${ }^{1}$ \\ Ian A Maher, ${ }^{6}$ Joseph F Sobanko, ${ }^{7,8}$ Todd V Cartee, ${ }^{9}$ Murad Alam (1) $1,10,11,12$
}

To cite: Ibrahim SA, Kang BY, Schlessinger DI, et al. Protocol for development of a core outcome set for clinical trials in melasma. BMJ Open 2022;12:e046953. doi:10.1136/ bmjopen-2020-046953

- Prepublication history for this paper is available online. To view these files, please visit the journal online (http://dx.doi org/10.1136/bmjopen-2020046953).

Received 13 November 2020 Accepted 09 January 2022

Check for updates

(C) Author(s) (or their employer(s)) 2022. Re-use permitted under CC BY-NC. No commercial re-use. See rights and permissions. Published by BMJ.

For numbered affiliations see end of article.

Correspondence to

Dr Murad Alam;

m-alam@northwestern.edu

\section{ABSTRACT}

Introduction Melasma is a pigmentation disorder of the skin. Characterised by brown to gray-brown patches on the face and neck, the condition predominantly affects women and has been associated with pregnancy, hormonal variation and sun exposure. Melasma can be disfiguring and anxiety-provoking, and quality of life is often adversely impacted. Management includes sun protection, laser and energy device therapy, topical and oral skin-bleaching agents and chemical peels. While clinical trials of melasma exist, there is a lack of consistency in reported outcomes, which has been a barrier to the aggregation of data in systematic reviews and meta-analyses. This protocol describes a planned process for development of a minimum set of outcomes (ie, 'core outcome set') that should be measured in all clinical trials of melasma.

Methods and analysis An exhaustive list of potential outcomes will be extracted from four sources: (1) systematic literature review of outcomes in clinical trials; (2) semistructured patient interviews; (3) brochures, pamphlets, clinical trial registries, and other published and unpublished sources and documentation; and (4) interviews with non-patient, non-physician stakeholders, including federal regulators, industry scientists and nonphysician providers. An international two-round Delphi process will then be performed to identify the outcomes deemed most important to patients and physicians. Subsequently, a consensus meeting will be convened to review and process the results, and to vote on a final set of core outcomes.

Ethics and dissemination Ethics approval was provided by the Northwestern University Institutional Review Board (protocol ID: STU00201637). This study is registered with both the Core Outcome Measures in Effectiveness Trials and Cochrane Skin-Core Outcome Set Initiative initiatives, and this protocol is in accordance with the guidelines for protocol development of both groups. All findings from the study described in this protocol will be disseminated to all stakeholders involved in the development process and will be submitted for publication in peer-reviewed journals. PROSPERO registration number CRD42020214189.

\section{INTRODUCTION}

Melasma is a chronic hyperpigmentation disorder primarily occurring in women. ${ }^{12}$ The
Strengths and limitations of this study

- This protocol describes a planned process for the development of a minimum set of outcomes (ie, 'core outcome set (COS)') that should be measured in all clinical trials of melasma.

- A long list of potential outcomes will be extracted from a systematic literature review, semistructured interviews, brochures and pamphlets, clinical trial registries and other published and unpublished sources and documentation.

- An international group of stakeholders, including patients, physicians, federal regulators, industry scientists, pharmacologists and pharmacists, nurses and non-physician providers will be included in the process.

- At least two rounds of Delphi process will then be performed to identify a provisional list of outcomes meeting a $70 \%$ consensus level for patient and physicians, followed by the convening of a consensus meeting to review and process the results, and to vote on a final set of core outcomes.

- This COS will establish 'what' should be measured, but not 'how' or 'when,' which will be defined in later development of core outcome measure set for melasma.

condition is characterised by brown, irregularly shaped macules and patches, commonly of the bilateral upper cheeks, mid forehead and upper lip. Predisposing risk factors for the development of melasma include darker skin types III and IV, genetic predisposition, ultraviolet radiation and hormonal changes due to pregnancy, menopause or medications. ${ }^{3-5}$ However, melasma remains a poorly understood condition that also arises in the absence of traditional risk factors, with a significant minority of cases occurring in men. ${ }^{6}$ Histologically, there is an increase of melanocytes and solar elastosis in the epidermis of melasma lesions compared with normal skin. ${ }^{247}$ Due to its sometimes striking impact on cosmetic appearance, melasma can 
cause psychological distress, thereby negatively affecting quality of life (QoL). ${ }^{3}$

Melasma is typically divided into three subtypes (epidermal, dermal or mixed) and can be classified via Wood's Lamp examination. Severity of lesions and area of involvement can be assessed using validated or more ad hoc measurement tools. Melasma has been treated with various modalities, including lasers and lights, chemical peels, skin-bleaching agents, such as hydroquinone, or oral agents, like tranexamic acid. ${ }^{18}$ However, current treatments are of limited efficacy and recurrence is the norm. Additionally, extant studies seldom assess patientreported outcomes, which are particularly relevant given the disfiguring nature of melasma.

Systematic reviews of treatments for melasma are limited in utility by the lack of standardisation in outcomes across trials. ${ }^{8}$ The selective inclusion of outcomes in publications, so-called selective outcome reporting bias, remains a problem in the reporting of clinical trials. In particular, the heterogeneity of outcomes reported across trials may affect the recommendations and conclusions of systematic reviews. ${ }^{9}$ In order to address the heterogeneity of outcomes in clinical trials of the same disease or condition, The Core Outcome Measures in Effectiveness Trials (COMET) initiative was created, with the goal of providing methodological support to facilitate development of standardised core outcome sets (COS) to be measured in health-related research. ${ }^{10}$ A COS is defined as a consensus-derived set of outcomes that are measured at minimum in all clinical studies of a given condition or disease. Similarly, another group, the Cochrane SkinCore Outcome Set Initiative (CS-COUSIN), was developed specifically to address COSs in dermatology. ${ }^{11}$ CS-COUSIN provides methodological support, and much of its approach is based on the experience of the Harmonising Outcome Measures for Eczema (HOME) initiative. $^{12-16}$

To date, there has been no COS published specifically for melasma. The data obtained from the investigation described in this protocol will define the minimum set of outcomes that should be reported in future clinical trials of melasma interventions.

\section{Objective}

The aim of this study will be to develop a COS through an international consensus process, for use in future clinical trials of melasma. The objective is to determine what outcomes should be reported as a minimum in future clinical trials of melasma.

\section{Scope of this COS}

This COS is envisioned as the global standard for all clinical trials examining the efficacy and safety of all melasma interventions, including both early and late phase trials. The COS to be developed is intended to apply to all individuals with melasma, regardless of age, gender and ethnicity.
This COS will establish 'what' should be measured, but not 'how' or 'when,' which will be defined in a later consensus study specific to outcome measures.

\section{METHODS AND ANALYSIS}

This study was designed using guidance provided by the CS-COUSIN and COMET initiatives and has been registered with both organisations. ${ }^{17-20}$ Additional guidance was provided by the HOME roadmap. ${ }^{16}$ The reporting of this protocol conforms to the The Core Outcome Set-STAndardised Protocol Items Statement checklist and the CS-COUSIN Core Domain Development Process guidance. ${ }^{18} 21$ This protocol is also based on prior work in protocol development by the Measurement of Priority Outcome Variables in Dermatologic Surgery (IMPROVED) Group, a COS development organisation for dermatological surgery-related conditions. ${ }^{22}$

\section{Study oversight}

The international study steering committee developing this COS will include four physicians (MA, IAM, JFS and TVC) as well as a patient representative. The latter, who will also have melasma, will represent others with this condition by providing input at key points to ensure that the patient perspective is incorporated. The four physicians have prior experience in developing COSs in dermatology, and therefore, also act as researchers in COS development. The steering committee will lead each stage of COS development and ensure methodological quality throughout the study. In addition, an independent member of the CS-COUSIN Methods Group (JJK) will provide guidance on the most current methodological recommendations for COS development.

\section{Study design}

\section{Identification of outcomes}

A long list of outcomes will be generated from four sources. First, a systematic review of the literature, which has been registered prospectively with the International Prospective Register of Systematic Reviews (PROSPERO, CRD42020214189), will be performed to identify and extract outcomes measured in randomised controlled trials of melasma. Specifically, with the help of a medical librarian, PubMed/Medline and Embase will be searched for the period 2006-2016 to detect English language human RCTs including, but not limited to, the following terms: [(melasma [title/abstract]) AND (randomized controlled trial [publication type]) AND (treatment OR therapy OR therapeutics)]. RCTs will be used to identify outcomes of interest, since it is usual and customary in COS methodology to focus on RCTs when they are available in sufficient variety and quantity. ${ }^{23-26}$ Inclusion criteria will be studies that: (1) are randomised and controlled; (2) assess the efficacy and/or safety of one or more interventions for treatment of melasma; (3) are available in the English language and (4) and involve human subjects. Articles will be excluded if they: 
(1) were published as a poster or conference abstract; or (2) the full text of the article is unavailable. Articles will be independently screened for eligibility by two investigators, and disagreements will be resolved by a third investigator. Two independent reviewers will then extract outcomes from individual studies. During extraction, QoL outcomes will be separated into distinct categories to ensure all of the various components of QoL that have been measured in previous investigations are included as possible core outcomes. Outcome measures will also be extracted during this step, and this data will be recorded for the future development of a core outcome measure set for melasma. ${ }^{27}$ The results of the systematic review will be published separately from the COS.

Second, other printed and electronic sources, including clinical trial registries (ClinicalTrials.gov searched for 'melasma', 2017-2021, no exclusion criteria), patient pamphlets (American Academy of Dermatology website searched for 'melasma', with inclusion criteria being 'all patient education material', and no exclusion criteria.) medical society brochures and relevant US Food and Drug Administration/European Medicines Agency guidance documents, will be reviewed to identify any additional outcomes not detected in the systematic review. Third, outcomes valued by patients will be identified by conducting semistructured interviews with patients diagnosed with melasma. These interviews will be audio recorded, transcribed and analysed by the methods of qualitative research to find outcomes considered relevant by patients. Fourth, semistructured interviews will be performed to identify any remaining outcomes deemed relevant by representatives of key non-physician, non-patient stakeholder classes, including industry scientists, (Leaders at a purposive sample of large, medium-sized and small US drug, device, and cosmetic companies involved in research on products for melasma will be contacted to ask for identification of qualified industry scientists in their employ who can help identify additional outcomes. In total, up to 20 industry scientists will be contacted. Drug and device safety regulators from the countries most represented in the systematic review will be contacted for interviews. When their names are publicly available, officials from dermatology or cosmeticrelated offices within these regulatory agencies will be contacted first). pharmacologists and pharmacists, drug and device safety regulators, (US Food and Drug Administration, European Medicines Agency, Ministry of Food and Drug Safety (Korean), Pharmaceuticals and Medical Devices Agency (Japan), Health Canada, Brazilian Health Regulatory Agency (ANVISA)), nurses and physician assistants. Semistructured interviews with patients and other stakeholders will be conducted by investigators who have been trained in this qualitative research technique. Specifically, such interviews will be comprised of a series of open-ended questions, followed by pre-established prompts, in the event that respondents are unclear as to the primary question. At the end of the semistructured interview, stakeholders will be asked to volunteer any additional information about the topic that they may wish to share. Interviewers will be strictly prohibited from using off-script leading questions that may bias data collection. After the semistructured interviews are completed, they will be transcribed, and the iterative methods of qualitative methods will be used to extract common themes. These themes, if not already present in the list of outcomes, will then be used to create new outcomes that will be appended to the long list. Nonphysician, non-patient stakeholders will not be invited to participate in the subsequent Delphi process but will be invited to the final consensus meeting.

\section{Final review of long list of outcomes}

The outcomes obtained from the sources above will be collated into a long list of provisional outcomes. Members of the steering committee will review and condense this list, eliminating duplicate items and combining items when possible, without loss of content. In accordance with the proposed definition of a unique outcome by Young et al, unique outcomes (ie, outcomes with 'original meaning and context') will be preserved, and other outcomes (ie, those "with different words, phrasing or spelling addressing the same concept and context') will be lumped together. ${ }^{28}$ The list of outcomes will then be placed into appropriate domains by two steering committee members using the COMET and CS-COUSIN taxonomies. ${ }^{29}{ }^{30}$ Lay definitions will be appended to all outcomes and reviewed by the melasma steering group patient representative to assure that patient stakeholders can actively participate in the forthcoming Delphi consensus process.

\section{Delphi participants}

Two separate groups, consisting of physicians and patients, respectively, will be invited to take part in the Delphi process. A global context will be provided by inviting physicians from the USA and from other countries on various continents, including a range of ethnicities. To include the perspective of researchers, the senior authors of all clinical trials extracted in our literature review will be included in the physician group. Eligible physician stakeholders will include dermatologists, clinical researchers, primary care providers and other medical specialists who have experience treating melasma. Demographic information, including participants' ethnicity, gender and specialty will be recorded. To account for potential dropouts, at least 100 physicians meeting any of the following criteria will be invited: corresponding author of a clinical trial of melasma included in our systematic review; among the most frequently published authors on melasma treatment, as identified through electronic databases; recent lecturers on the topic of melasma at national or international dermatology professional society meetings in any country; or members of a national or international dermatological society (Representative board members of the following societies will be invited to participate as individuals in the 
Delphi to ensure inclusion of the perspectives of expert clinicians and researchers who may not have recently published in the literature: American Academy of Dermatology Association; American Society for Laser Medicine and Surgery; African Society of Dermatology and Venereology; Asian Academy of Dermatology and Venereology; Arab Academy of Dermatology and Aesthetics; Argentine Society of Dermatology; Brazilian Society of Dermatology; British Association of Dermatologists; Canadian Dermatology Association; European Academy of Dermatology and Venereology; French Society of Dermatology; Mexican Society of Dermatology; Skin of Colour Society; World Congress of Dermatology). with clinical expertise in melasma treatment, as demonstrated by committee or other affiliations.

Physicians who agree to participate will be asked to identify one or more melasma patients who may be invited to join the patient Delphi group, with a goal of 15 patient stakeholders participating in the Delphi. All recruitment will be done by our study team and will be approved by our ethics committee. However, this will not entail limiting patient recruitment from our site only, since we will be asking physician Delphi participants located elsewhere to volunteer patients who may choose to participate in the study. Such patient volunteers will contact the research staff at our site, who will consent and enroll them, if appropriate. Additional methods will be taken to ensure patient involvement throughout the study, including: (1) specifying patient involvement in the Institutional Review Board (IRB) protocol; (2) seeking relevant input from patients; (3) maintenance of investigator open-mindedness to the patient perspective; (4) careful reviewing of all outcomes with patient representatives; (5) thorough note taking; (6) taking time to reflect on patient feedback and (7) identifying and engaging a diverse group of patient participants. ${ }^{31}$

\section{Modified Delphi process}

From the long list of potential outcomes vetted by the steering committee, a core set of outcomes will be provisionally selected by stakeholders through a Delphi process, as recommended by the COMET and CS-COUSIN initiatives. ${ }^{17} 18{ }^{30}$ Specifically, each Delphi participant will be asked to rate each outcome for its level of importance on a scale from 1 to 9 . Average ratings for each outcome, and relevant participant comments, will then be redistributed to each survey participant, who will have the option of changing his or her earlier ratings based on the additional information surfaced in this process. Prior to a consensus meeting, at least two Delphi rounds will be conducted.

\section{Delphi rounds}

During each Delphi round, the provisional outcomes in the long list will be presented to each participant for rating. Participants will rate each outcome on a 9-point scale developed by the Grading of Recommendations Assessment, Development and Evaluation working group, with ' 9 ' denoting 'critically important' 'and ' 1 ,' 'not that important. ${ }^{32}$ In each round, each participant will have the option to select ' 10 ' if they are uncertain about an outcome's need for inclusion. Also in each round, each participant will have the option to identify new outcomes that they feel should be added in the subsequent round. All previously included outcomes will be carried to the next round. Participants will have 3 weeks to complete each Delphi round, and will receive weekly reminders until they do, or time expires.

Results from round 1 will be analysed by outcome and for each stakeholder group. Then, round 2 will commence. In round 2, participants will be graphically shown the distribution of scores for each item for each stakeholder group from round 1 , and also their own individual ratings for each outcome from the previous round, and asked to score each item again. New outcomes will be added to round 2 if suggested by two or more participants in round 1 , if the steering committee determines the suggested outcome(s) to be unique from existing outcomes. $^{28}$

Summarised scores from round 2, analysed by outcome and for each stakeholder group, will be presented at the consensus meeting. Attrition is possible between Delphi rounds, and although numeric data (eg, mean, median and range of scores) from round 2 alone will be analysed and presented at the consensus meeting, written feedback from both rounds will be collated and discussed at the consensus meeting, as well.

\section{Definition of provisional consensus}

Outcomes will be retained in the provisional consensus pool if $70 \%$ of the participants score 7,8 or 9 with less than $15 \%$ scoring $1-3 .^{33}$ Outcomes will be removed from the provisional consensus pool if $70 \%$ or more of the participants score 1,2 or 3 and less than $15 \%$ score $7-9$. To avoid having a COS that entails too many items, if the provisional list of included outcomes is longer than expected, participants at the consensus meeting will be urged to further refine and abbreviate this list. The definition of consensus is based on previous, published COS consensus methodology and guidance of the COMET Methodology Group. ${ }^{174-36}$ Outcomes that have not reached consensus will also be retained for discussion during the consensus meeting.

\section{Consensus meeting}

A series of virtual consensus meetings will be held to discuss the results of the Delphi, to review the provisional COS as well as the outcomes for which consensus has not been reached, and to move towards selection of a final COS. The reason to have more than one consensus meeting is to avoid the scenario in which the loudest voices dominate, and patients in particular are not heard as clearly and to the extent that they should be. Since we anticipate 30-60 healthcare professionals, and approximately five patients to participate in the process, we anticipate three virtual consensus meetings of 15-20 participants each, with each meeting also including patient participants. An additional benefit of having multiplate consensus 
meetings is that different schedules and time zones can be accommodated. Finally, if the outcomes of the different consensus meetings are not fully consistent, an email ballot will be sent to all participants individually to resolve any remaining issues. Each meeting will be moderated by an independent facilitator, and invited participants will include all physicians and patients who participated in at least the first round of the Delphi. Each meeting will have balanced representation across stakeholder groups and geographical regions to ensure the result is development of a global COS. Other non-physician, non-patient stakeholders will be invited, as well.

Informed by the Delphi results, feedback regarding the consensus-derived set of provisional outcomes and outcomes for which consensus has not been reached will be elicited from the consensus meeting participants with the assistance of the facilitator. Using live polling software, participants will vote to include or not include outcomes into the final core set of outcomes. If multiple consensus meetings are held, and if there is any inconsistency between the outcomes selected in these, a final email ballot will be circulated to all consensus meeting participants to confirm the final COS. The result will be a COS that reflects the priorities and concerns of all stakeholders.

\section{Timeline}

The expected timeline from the start of the study to full development of the core set of outcome domains will be 18-24 months. Identification of an initial list of outcomes, via systematic review followed by qualitative interviews, will span approximately $7-8$ months. An additional seven to ten months will be dedicated to conducting the Delphi survey and convening the consensus meeting, followed by approximately $4-6$ months for analysing feedback and drafting, circulating and finalising the manuscript.

\section{Patient and public involvement}

The patient and public perspective will be sought at multiple points in this study. Patient stakeholders will review plain language summaries of outcome definitions. A minimum of one patient representative will be included in the research team, as described earlier in this protocol. Additionally, patients will be recognised as key stakeholders during the identification and prioritisation of outcomes, with fully one-half of the Delphi process reserved for patients. Patients will be encouraged to provide feedback before (semistructured interviews), during and after (at the consensus meeting) the Delphi process to ensure that patient-centred outcomes are incorporated. Lastly, with their consent, patient representatives will be named as contributors in any published work that arises from the study.

\section{ETHICS AND DISSEMINATION}

\section{Dissemination and implementation of results}

The full development of this COS and the results of the study will be reported in peer-reviewed journals. The main results of the study, including the COS, will be disseminated to all participants through email at the time of study publication. Researchers will be encouraged to use the COS when performing future trials.

\section{Ethical approval and consent to participate}

Ethical approval and consent to participate for the study has been granted from the Northwestern University IRB (protocol ID: STU00201637). Informed consent will be presented before registering for the Delphi. The Northwestern University IRB has waived written informed consent and has approved verbal consent for interviews, and online consent for the Delphi process.

\section{DISCUSSION}

Despite the numerous completed and ongoing clinical trials of treatments for melasma, there is currently no COS informing such investigations. The proposed COS for melasma would provide a minimum set of outcomes to be reported in all trials of melasma, thus standardising future outcomes reporting. Investigators would be free to consider and include additional outcomes beyond the core set, but their use of at least the core set would allow aggregation and comparison of data across melasma trials. Cross-trial comparisons of treatments and largescale meta-analyses would, in turn, enable more definitive conclusions on the merits of available treatments.

\section{Trial registration and status}

This study has been registered with both the COMET and CS-COUSIN initiatives for COS development, and the development of this protocol is in accordance with the guidelines for protocol development of both groups. The development of the COS is currently in its initial phase of outcome extraction.

\section{Author affiliations}

${ }^{1}$ Department of Dermatology, Northwestern University Feinberg School of Medicine, Chicago, Illinois, USA

${ }^{2}$ Division of Dermatology, Department of Internal Medicine, Washington University in St Louis, St Louis, Missouri, USA

${ }^{3}$ Department of Dermatology and Cutaneous Surgery, University of Miami Miller School of Medicine, Miami, Florida, USA

${ }^{4}$ Centre for Biostatistics, Manchester Academic Health Science Centre, University of Manchester, Manchester, UK

${ }^{5}$ Centre for Evidence-Based Healthcare, Medical Faculty Carl Gustav

Carus, Technical University Dresden, Dresden, Germany

${ }^{6}$ Department of Dermatology, University of Minnesota, Minneapolis, Minnesota, USA ${ }^{7}$ Department of Dermatology, University of Pennsylvania, Philadelphia, Pennsylvania, USA

${ }^{8}$ Division of Dermatologic Surgery, University of Pennsylvania, Philadelphia, Pennsylvania, USA

${ }^{9}$ Department of Dermatology, Penn State Health, Hershey, Pennsylvania, USA ${ }^{10}$ Department of Otolaryngology, Feinberg School of Medicine, Northwestern University, Chicago, Illinois, USA

${ }^{11}$ Department of Surgery, Feinberg School of Medicine, Northwestern University, Chicago, Illinois, USA

${ }^{12}$ Department of Medical Social Sciences, Feinberg School of Medicine, Northwestern University, Chicago, Illinois, USA

Contributors Study concept and design: SAI, BYK, DIS, JCT, JJK, JS, EP, IAM, JFS, TVC and MA. Drafting of the manuscript: SAI, BYK, SGC, EP, TVC and MA. Critical 
revision of the manuscript for important intellectual content: SAI, BYK, DIS, JJK, EP and MA. Obtained funding: MA. Study supervision: MA. All authors read and approved the final manuscript.

Funding This publication was supported by Merz Centre for Quality and Outcomes Research in Dermatologic Surgery and the IMPROVED (Measurement of Priority Outcome Variables in Dermatologic Surgery) Group.

Competing interests None declared.

Patient and public involvement Patients and/or the public were involved in the design, or conduct, or reporting, or dissemination plans of this research. Refer to the Methods section for further details.

Patient consent for publication Not applicable.

Provenance and peer review Not commissioned; externally peer reviewed.

Open access This is an open access article distributed in accordance with the Creative Commons Attribution Non Commercial (CC BY-NC 4.0) license, which permits others to distribute, remix, adapt, build upon this work non-commercially, and license their derivative works on different terms, provided the original work is properly cited, appropriate credit is given, any changes made indicated, and the use is non-commercial. See: http://creativecommons.org/licenses/by-nc/4.0/.

\section{ORCID iDs}

Jamie J Kirkham http://orcid.org/0000-0003-2579-9325

Murad Alam http://orcid.org/0000-0001-8752-3821

\section{REFERENCES}

1 Kauh YC, Melasma ZTF. Rheumaderm. advances in experimental medicine and biology. . Springer, 1999: Vol 455. 491-9.

2 Kang WH, Yoon KH, Lee E-S, et al. Melasma: histopathological characteristics in 56 Korean patients. $\mathrm{Br} \mathrm{J}$ Dermatol 2002;146:228-37.

3 Balkrishnan R, McMichael AJ, Camacho FT, et al. Development and validation of a health-related quality of life instrument for women with melasma. Br J Dermatol 2003;149:572-7.

4 Mahmoud BH, Ruvolo E, Hexsel CL, et al. Impact of long-wavelength UVA and visible light on melanocompetent skin. J Invest Dermatol 2010;130:2092-7.

5 Ortonne JP, Arellano I, Berneburg M, et al. A global survey of the role of ultraviolet radiation and hormonal influences in the development of melasma. J Eur Acad Dermatol Venereol 2009;23:1254-62.

6 Jang YH, Sim JH, Kang HY, et al. The histopathological characteristics of male melasma: comparison with female melasma and lentigo. J Am Acad Dermatol 2012;66:642-9.

7 Hernández-Barrera R, Torres-Alvarez B, Castanedo-Cazares JP, et al. Solar elastosis and presence of mast cells as key features in the pathogenesis of melasma. Clin Exp Dermatol 2008;33:305-8.

8 Rajaratnam R, Halpern J, Salim A, et al. Interventions for melasma. Cochrane Database Syst Rev 2010;7:CD003583.

9 Kirkham JJ, Dwan KM, Altman DG, et al. The impact of outcome reporting bias in randomised controlled trials on a cohort of systematic reviews. BMJ 2010;340:c365.

10 Comet initiative. Available: https://www.comet-initiative.org/ [Accessed 02 Oct 2020].

11 Cochrane skin - core outcome set initiative. Available: http://cscousin.org/ [Accessed 02 Oct 2020].

12 Schmitt J, Spuls P, Boers M, et al. Towards global consensus on outcome measures for atopic eczema research: results of the home II meeting. Allergy 2012;67:1111-7.

13 Schmitt J, Williams H, HOME Development Group. Harmonising outcome measures for eczema (home). Report from the first international consensus meeting (home 1), 24 July 2010, Munich, Germany. Br J Dermatol 2010;163:1166-8.

14 Schmitt J, Spuls PI, Thomas KS, et al. The Harmonising outcome measures for eczema (home) statement to assess clinical signs of atopic eczema in trials. J Allergy Clin Immunol 2014;134:800-7.
15 Chalmers JR, Schmitt J, Apfelbacher C, et al. Report from the third International consensus meeting to harmonise core outcome measures for atopic eczema/dermatitis clinical trials (home). $\mathrm{Br} \mathrm{J}$ Dermatol 2014;171:1318-25.

16 Schmitt J, Apfelbacher C, Spuls PI, et al. The Harmonizing outcome measures for eczema (home) roadmap: a methodological framework to develop core sets of outcome measurements in dermatology. $J$ Invest Dermatol 2015;135:24-30.

17 Williamson PR, Altman DG, Bagley H, et al. The comet Handbook: version 1.0. Trials 2017;18.

18 CS-COUSIN Methods Group. Guidance on how to develop a core outcome set for skin disease by the CS-COUSIN methods group, version 2.2, 2018.

19 Comet initiative | core outcome set for Melasma, 2020. Available: https://www.comet-initiative.org/studies/details/752 [Accessed 29 Oct 2020].

20 Core outcome set for Melasma. Available: http://cs-cousin.org/ melasma/ [Accessed 29 Oct 2020].

21 Kirkham JJ, Gorst S, Altman DG, et al. Core outcome SetSTAndardised protocol items: the COS-STAP statement. Trials 2019;20:116.

22 lyengar S, Williamson PR, Schmitt J, et al. Development of a core outcome set for clinical trials in rosacea: study protocol for a systematic review of the literature and identification of a core outcome set using a Delphi survey. Trials 2016;17:429.

23 Reynolds KA, Schlessinger DI, Vasic J, et al. Core outcome set for actinic keratosis clinical trials. JAMA Dermatol 2020;156:326-33.

24 Reynolds KA, Schlessinger DI, Yanes AF, et al. Development of a core outcome set for cutaneous squamous cell carcinoma trials: identification of core domains and outcomes. $\mathrm{Br} J$ Dermatol 2021;184:1113-22

25 Ingram JR, Hadjieconomou S, Piguet V. Development of core outcome sets in hidradenitis suppurativa: systematic review of outcome measure instruments to inform the process. $\mathrm{Br} J$ Dermatol 2016;175:263-72.

26 Thorlacius L, Ingram JR, Garg A, et al. Protocol for the development of a core domain set for hidradenitis suppurativa trial outcomes. BMJ Open 2017;7:14733.

27 Guideline for selecting instruments for a core outcome set • COSMIN. Available: https://www.cosmin.nl/tools/guideline-selectingproms-cos/ [Accessed 02 Oct 2020].

28 Young AE, Brookes ST, Avery KNL, et al. A systematic review of core outcome set development studies demonstrates difficulties in defining unique outcomes. J Clin Epidemiol 2019;115:14-24.

29 Comet initiative | outcome classification. Available: https://www. comet-initiative.org/Resources/OutcomeClassification [Accessed 02 Oct 2020].

30 Lange T, Kottner J, Weberschock T, et al. Outcome assessment in dermatology clinical trials and Cochrane reviews: call for a dermatology-specific outcome taxonomy. J Eur Acad Dermatol Venereol 2021;35:523-35.

31 Smith $\mathrm{H}$, Horobin A, Fackrell K, et al. Defining and evaluating novel procedures for involving patients in core outcome set research: creating a meaningful long list of candidate outcome domains. Res Involv Engagem 2018;4:1-12.

32 Guyatt GH, Oxman AD, Kunz R, et al. Grade guidelines: 2. framing the question and deciding on important outcomes. J Clin Epidemiol 2011;64:395-400.

33 Harman NL, Bruce IA, Kirkham JJ, et al. The importance of integration of stakeholder views in core outcome set development: otitis media with effusion in children with cleft palate. PLoS One 2015;10:e0129514

34 Williamson PR, Altman DG, Blazeby JM, et al. Developing core outcome sets for clinical trials: issues to consider. Trials 2012;13:132.

35 Potter S, Holcombe C, Ward JA, et al. Development of a core outcome set for research and audit studies in reconstructive breast surgery. Br J Surg 2015;102:1360-71.

36 Blazeby JM, Macefield R, Blencowe NS, et al. Core information set for oesophageal cancer surgery. Br J Surg 2015;102:936-43. 